\title{
Defect formation and diffusion mechanism in ion-assisted molecular-beam epitaxy
}

\author{
C. J. Tsai, ${ }^{*}$ T. Vreeland, Jr., and H. A. Atwater \\ Thomas J. Watson Laboratory of Applied Physics, California Institute of Technology, Pasadena, California 91125
}

(Received 25 November 1991)

\begin{abstract}
A simple moving boundary diffusion model has been used to characterize defect incorporation kinetics during ion-assisted molecular-beam epitaxy. The model permits analysis of the dependence of the final defect concentration on the growth rate, defect diffusivity, defect production range, and the shape of defect depth distribution. The results indicate a linear dependence of the final defect concentration on the ion-to-atom flux ratio which is in the growth-rate-limited regime of the model. Comparison between the model and the film strains measured by $x$-ray rocking curve analyses has been made and reveals that the thermal spike energy deposited by the bombarding ions during epitaxial growth has a significant effect on the apparent activation energy of the defect migration. A transition temperature above which the defect migration is thermally activated and below which the defect migration is cascade assisted can be defined. The experimentally observed temperature dependence of the defect concentration can be attributed to cascade-assisted diffusion of the defects. Comparison between the model and the multisite multiply activated migration model for low-energy dopant incorporation has also been made. The results show the similarity between the defect incorporation and dopant incorporation which gives a unified view of both processes.
\end{abstract}

\section{INTRODUCTION}

Low-energy ion beams have been used as an extra control element for semiconductor processing. The applications include sputtering, surface cleaning, enhanced dopant incorporation, ${ }^{1}$ surface smoothing, ${ }^{2-5}$ growth mode modification, ${ }^{6}$ and strain modification. ${ }^{7,8}$ Direct low-energy ion-beam deposition, ${ }^{9}$ partially-ionized vapor deposition, ${ }^{10}$ ion-beam sputter deposition, ${ }^{11,12}$ and concurrent low-energy ion bombardment during sputtering deposition ${ }^{13}$ also produced $\mathrm{Si}$ homoepitaxial films at much lower growth temperature compared to the conventional thermal molecular-beam epitaxy (MBE).

During low-energy ion bombardment, injection of pointlike defects into the subsurface region results in entrapment of defects. When low-energy ion bombardment is combined with epitaxial growth at a constant rate, the resulting uniform dispersion of pointlike defects leads to uniformly strained epitaxial layers. The linear dependence of the strain on the ion-to-atom flux ratio ${ }^{7,8}$ suggests that defect recombination is consistent with firstorder kinetics and that the major sink for defect annihilation is the free surface. Following these assumptions, we can conceptualize the steady-state defect concentration as a simple moving boundary diffusion problem using a continuum approximation. The activation processes for defect diffusion were explained via a cascade-assisted diffusion model which is activated by the thermal spike energy deposited by the bombarding ions. A multisite multiply activated migration model of low-energy ionbeam-enhanced dopant incorporation using surface, bulk, and three intermediate sites with different activation barriers has been proposed by Ni et al. ${ }^{14}$ A qualitative comparison of the present work with this model was also discussed.

\section{EXPERIMENT}

Experiments were done in a custom-designed molecular-beam epitaxy system with a base pressure of $1 \times 10^{-10}$ Torr. The system is equipped with two electron guns for deposition of $\mathrm{Si}$ and $\mathrm{Ge}$, a Kaufmann-type ion source capable of producing ions at energies of 50-1500 $\mathrm{eV}$, and a reflection high-energy electron-diffraction (RHEED) apparatus. Strain-modified films of $100 \mathrm{~nm}$ were grown at rates which ranged from 0.1 to $0.7 \mathrm{~nm} / \mathrm{s}$ on (001) Ge substrates with concurrent ion-beam bombardment. The growth conditions including ion species, growth rate, and ion flux are listed in Table I. RHEED was used for in situ monitoring of the surface. A fourcrystal high-resolution x-ray diffractometer ${ }^{15}$ using a $\mathrm{Cu}$ $K_{\alpha 1}$ radiation source was employed to analyze the film strains. Comparison of experimental data with simula-

TABLE I. Growth conditions of IAMBE Ge films on $\mathrm{Ge}(001)$ substrate with concurrent $200-\mathrm{eV} \mathrm{Ar}^{+}$ion beams and $200-\mathrm{eV} \mathrm{Xe}^{+}$ion beams at $300^{\circ} \mathrm{C}$.

\begin{tabular}{ccccc}
\hline $\begin{array}{c}\text { Growth rate } \\
(\mathrm{nm} / \mathrm{s})\end{array}$ & $\begin{array}{c}\text { Ion flux } \\
\mu \mathrm{A} / \mathrm{cm}^{2}\end{array}$ & Ions/atoms & $\begin{array}{c}\text { Strain } \\
(\%)\end{array}$ & Ions \\
\hline 0.60 & 4.20 & 0.010 & 0.20 & $\mathrm{Ar}^{+}$ \\
0.30 & 4.68 & 0.022 & 0.53 & $\mathrm{Ar}^{+}$ \\
0.35 & 6.45 & 0.026 & 0.95 & $\mathrm{Ar}^{+}$ \\
0.25 & 6.13 & 0.035 & 0.82 & $\mathrm{Ar}^{+}$ \\
0.24 & 7.58 & 0.045 & 1.57 & $\mathrm{Ar}^{+}$ \\
& & & & \\
0.30 & 4.84 & 0.023 & 0.17 & $\mathrm{Xe}^{+}$ \\
0.20 & 5.97 & 0.042 & 0.44 & $\mathrm{Xe}^{+}$ \\
0.17 & 8.06 & 0.067 & 0.29 & $\mathrm{Xe}^{+}$ \\
0.10 & 9.68 & 0.137 & 0.61 & $\mathrm{Xe}^{+}$ \\
\hline \hline
\end{tabular}


tions based on dynamical x-ray-diffraction theory ${ }^{16}$ was used to determine the strains.

\section{RESULTS AND DISCUSSIONS}

\section{A. IAMBE Growth}

\section{Moving boundary diffusion model}

We consider the growth surface to be a onedimensional system in a frame moving with velocity $v$, by substituting $x+v t$ for $x$ into the diffusion equation and assuming the diffusivity of the defects at the growth temperature $D$ is independent of concentration. We have

$$
\frac{d N}{d t}=D \frac{d^{2} N}{d x^{2}}-v \frac{d N}{d x}+S
$$

where $N$ is the defect concentration, $x$ is the distance measured from the moving growth surface, $v$ is the growth rate, and $S$ is the source function representing the defect profile generated by the ion beam. We are interested in the steady-state solution, $d N / d t=0$. Equation (1) is basically the same as the diffusion equation used by Myers-Beaghton ${ }^{17}$ for studying the diffusion and adatom interaction during epitaxial growth on a vicinal surface without a recombination term. We choose two simple source functions, (i) a step function and (ii) a Gaussian function, as shown in Fig. 1. The choice of the step function is to illustrate the functional dependence of the important parameters in the ion-assisted molecular-beam epitaxy (IAMBE) process. We define a projected defect production range $R_{p}$ for the step source function, above which the defect production rate can be ignored. In the Gaussian source function, $\Delta R_{p}$ is the standard deviation of the projected defect production range. The quantities $R_{p}$ and $\Delta R_{p}$ have the usual meaning when we deal with
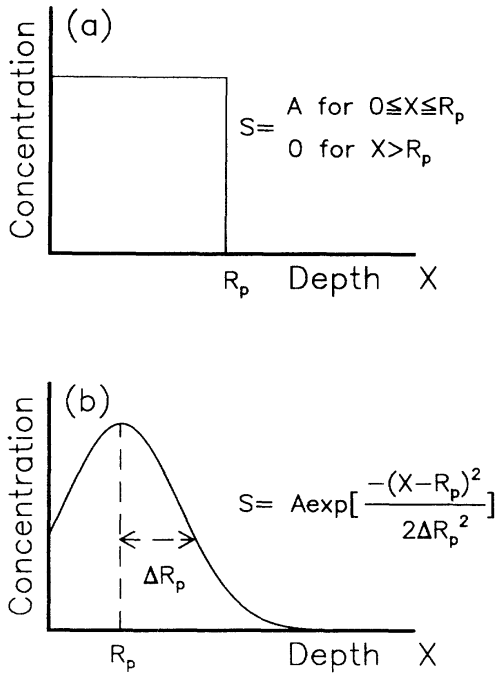

FIG. 1. Defect production profiles used for analytic solution of Eq. (1) with total defects per uit area per unit time. (a) A step function with defect production range $R_{p}$, (b) a Gaussian with defect production range $R_{p}$ and standard range deviation $\Delta R_{p}$.

the dopant incorporation, i.e., $R_{p}$ is the projected range and $\Delta R_{p}$ is the range straggling. We also define $\alpha=v / D$. For purposes of comparison of these two source functions, we fixed the total defect flux (per unit surface area per unit time) and set it equal to $K$. The boundary conditions used in solving the problem for the step function are $N(0)=0, N(\infty)=C ; N$ is continuous at position $R_{p}$, and the slope of $N$ at position $R_{p}$ is also continuous and for the Gaussian function they are $N(0)=0, N(\infty)=C$, and the slope of $N$ at $x=\infty$ is 0 . The solutions for the final concentration in these two cases are the following:

(i) $C=\frac{K}{v}+\frac{K}{\alpha R_{p} v}\left(e^{-\alpha R_{p}}-1\right)$,

(ii) $C=\frac{K}{v}\left\{1-\frac{e^{-\alpha R_{p}+\alpha^{2} \Delta R_{p}^{2} / 2} \operatorname{erfc}\left(-\frac{R_{p}}{\sqrt{2} \Delta R_{p}}+\frac{\alpha \Delta R_{p}}{\sqrt{2}}\right)}{\left[1+\operatorname{erf}\left(\frac{R_{p}}{\sqrt{2} \Delta R_{p}}\right)\right]}\right\}$.

For the step defect distribution, the final defect concentrations in the growth-rate-limited regime, $\alpha R_{p} \gg 1$, are proportional to $K / v$, while in the diffusion-limited regime, $\alpha R_{p} \ll<1$, the final concentrations are proportional to $K R_{p} / D$. For the Gaussian defect distribution, the final concentration $C$ can be proved to be convergent and the criterion for achieving the growth-rate-limited regime depends on the value of $M=R_{p} / \sqrt{2} \Delta R_{p}$. The sufficient condition for the growth-rate-limited regime is $\alpha R_{p} \gg 2 M+M^{2}$, while for $M \gg>1$, the exponential term in Eq. (2ii) will dominate the approach of the final concentration to $K / v$, the condition becomes $\alpha R_{p} \gg 1$. The condition states that, for a fixed defect production range, broader defect production profiles require higher growth rates to trap all the defects. Note that $K$ is proportional to the ion flux, $J_{i}$. Figure 2 shows the final concentrations as a function of $\alpha R_{p}$ for the step function and the Gaussian functions with $M=0.707$ and 2.828. The final defect concentration of a step source function never reaches unity because a certain portion of the defects can be classified as surface defects. This clearly shows that, in the IAMBE processes, the definition of surface defects (defects that are generated in the substrate and escape out of the substrate), and bulk defects (defects that are gen- 


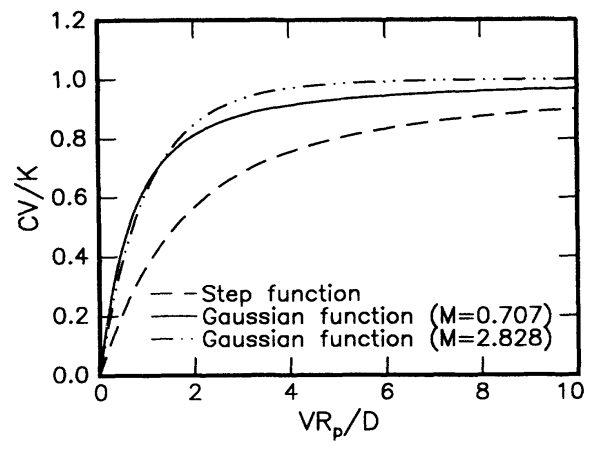

FIG. 2. The dependence of the final normalized defect concentrations on $\alpha R_{p}$ for a step source function, a Gaussian source function with $R_{p} / \sqrt{2} \Delta R_{p}=0.707$, and a Gaussian source function with $R_{p} / \sqrt{2} \Delta R_{p}=2.828$.

erated and trapped in the substrate), are not only related to the diffusivity of defects and the defect production profile but also to the growth velocity. Let us use the step-function case to illustrate the definition of the surface defects and bulk defects in the IAMBE process. The portion of bulk defects is defined as the ratio of the final retained defects per unit area to the defects generated per unit area by the ion beam. The total number of defects per unit area generated by the ion beam in the range of $0-R_{p}$ is $K R_{p} / v$ and the total number of final retained defects per unit area within the same range is $C R_{p}$; thus the portion of bulk defects is $C v / K$ and the portion of surface defects is $1-(C v / K)$. Thus, the curves in Fig. 2 also define the portion of bulk defects as a function of $\alpha R_{p}$.

To compare this model with experiment, we measured the strains of the films grown by IAMBE. A simple analysis of the strain modification was based on the assumption that the strains measured from the x-ray rocking curve analyses were proportional to the product of defect concentration and the defect-associated volume changes. Table I lists data associated with Ge films grown by the IAMBE processes. Figure 3 shows the dependence of the strains on the ion-to-atom flux ratio $\left(J_{i} / J_{a}\right)$. The ion-to-atom flux ratio, $J_{i} / J_{a}$, is equal to

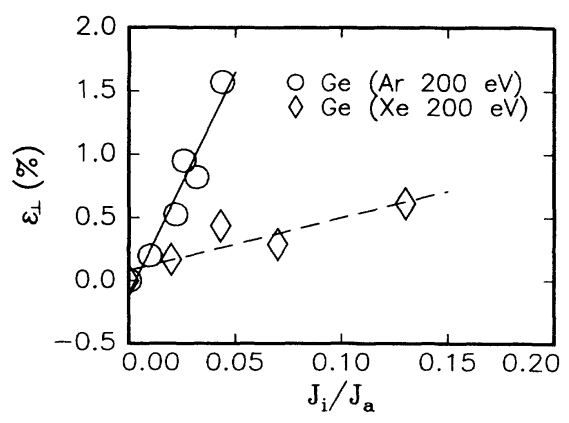

FIG. 3. The perpendicular strains of IAMBE Ge films with $200-\mathrm{eV} \mathrm{Ar}^{+}$bombardment and $200-\mathrm{eV} \mathrm{Xe}^{+}$bombardment at $300^{\circ} \mathrm{C}$, listed in Table $\mathrm{I}$ as a function of ion-to-atom ratio $\left(J_{i} / J_{a}\right)$. The solid and dashed lines are fits for the moving boundary diffusion model in the growth-rate-limited regime.
$K / v$ if one ion produces one defect. This is not necessary true for the case of defect incorporation. However, the ion-to-atom flux ratio is proportional to the value of $K / v$. Thus, for the case of defect incorporation, $J_{i} / J_{a}$ which is directly measurable was used. We note that in these growth conditions the strains are roughly proportional to the ion-to-atom flux ratio only, and not the absolute ion flux. This suggests that the growth conditions are in the growth rate-limited regime since the final concentration in this regime is proportional to $K / v$. We can find the upper limit of the defect diffusivity, taking the growth rate as $0.1 \mathrm{~nm} / \mathrm{s}$, the defect production range estimated from the TRIM calculation is on the order of $1 \mathrm{~nm}$ for the above ion-energy condition, thus, $D \ll 10^{-15} \mathrm{~cm}^{2} / \mathrm{s}$ for growth conditions to be in the growth-rate-limited regime. In previous experiments, we estimated an apparent activation energy for strain annihilation obtained from the dependence of the strains on the growth temperatures to be $Q=0.12 \mathrm{eV} .^{7}$ If we use this activation energy and put it into the condition $D \ll 10^{-15} \mathrm{~cm}^{2} / \mathrm{s}$, we find that the pre-exponential factor, at $600 \mathrm{~K}$, is $D_{0} \ll<10^{-14}$ $\mathrm{cm}^{2} / \mathrm{s}$. This value implies a diffusion site density which is too low to be consistent with ordinary bulk thermal diffusion, and thus suggests the apparent activation energy obtained from the growth temperature dependence is not the true activation energy for a single rate-limited bulk defect diffusion process. The estimated activation energy may instead reflect the thermal spike energy deposited by the bombarding ions. Thus, we will consider the temperature rise in the subsurface region of a film due to the energy deposited by the energetic ions.

\section{Cascade-assisted diffusion}

An energetic particle striking a target will generate a cascade. Cascade recovery consists in principle of two parts, (i) athermal recovery and (ii) thermal recovery. The athermal recovery is due to the fact that there are both vacancies and interstitials inside the cascade region. The thermal recovery of the cascade is due to the local heating (thermal spike) which provides thermal energy to the defects. The fact that (i) the defects left after the events of cascade recovery are stable at the growth temperature and (ii) nonetheless we measured a very low apparent activation energy suggests that a possible explanation for the apparent activation energy in the growth temperature dependence of the strain annihilation is related to cascade-assisted defect diffusion. The final defects generated by the ion beam have an activation energy for movement much larger than the apparent activation energy.

The thermal spike concept has been used in sputtering and ion mixing. Several attempts at calculation of the temperature evolution inside a cascade region ${ }^{18-20}$ have been made; we follow Vineyard's calculation of the temperature evolution inside a cylindrical thermal spike, ${ }^{20}$ which has been successfully applied to explain the ionbeam mixing data. ${ }^{21}$ It is important to remember the basic assumptions of the thermal spike model that the heat conduction equation is valid over the microscopic region and short time scale involved. The medium is con- 
sidered to have a thermal conductivity $\kappa$, a heat capacity $C_{h}$, and a density $\rho$. The major contribution of the spike-induced jumps of the defects is from the core region at the beginning of the time; thus, for simplicity and to a first-order approximation, we assume that the heat capacity and the thermal conductivity are temperature independent at high temperature. Consider the liberation of the heat per unit length, $F_{d}$, at a point at the origin at time, $t=0$, with the initial medium temperature equal to the substrate growth temperature $T_{g}$; the temperature $T(s, t)$ at position $s$ and time $t$ corresponding to the solution for the heat conduction equation is

$$
T(s, t)=\frac{F_{d}}{4 \pi \kappa t} \exp \left(-\frac{\rho C_{h} s^{2}}{4 \pi \kappa t}\right)+T_{g} .
$$

The number of jumps per unit time contributed from the spike for a defect with a migration activation energy $Q_{m}$ and an attempt frequency $v$ is

$$
\begin{aligned}
\eta=J_{i} v \int_{0}^{\infty} 2 \pi s d s \int_{t_{0}}^{\infty}\{ & \exp \left[-\frac{Q_{m}}{k_{B} T(r, t)}\right] \\
& \left.-\exp \left[-\frac{Q_{m}}{k_{B} T_{g}}\right]\right\} d t,
\end{aligned}
$$

where $J_{i}$ is the ion flux and $k_{B}$ is the Boltzmann constant. The initial width of the cylindrical spike is determined by the starting time $t_{0}$. This cylindrical spike is assumed to have influence on the subsurface region only. We performed numerical integration of Eq. (4) with parameters $F_{d}=150 \mathrm{eV} / \mathrm{nm}$ estimated from TRIM simulation of 200-eV $\mathrm{Ar}^{+}$-bombarded $\mathrm{Ge}, J_{i}=3 \times 10^{13} \mathrm{ions} / \mathrm{cm}^{2} \mathrm{~s}$, $C_{h \rho}=27 \mathrm{~J} / \mathrm{mole} \mathrm{K}$ is the heat capacity for liquid Ge taken from Ref. 22 , and $\kappa=0.014$ watt $/ \mathrm{cm} \mathrm{K}$, as suggested by Thompson and Nelson. ${ }^{23}$ The adjustable parameters are the activation energy for the defect migration and the starting time $t_{0}$, which corresponds to the initial distribution of the deposited energy ${ }^{18}$ or the time needed to establish the Maxwell-Boltzmann distribution, i.e., local thermal equilibrium, which ranges between $10^{-12}$ and $10^{-13} \mathrm{~s}^{24}$ The effective diffusivity can be defined from Eq. (4) as $D=D_{0}\left[\eta / v+\exp \left(-Q_{m} / K_{B} T_{g}\right)\right]$, where $D_{0}$ is the preexponential for thermal diffusion.

Figure 4 shows three curves of effective diffusivity with (i) $Q_{m}=1.5 \mathrm{eV}$ and $t_{0}=4 \times 10^{-13} \mathrm{~s}$, (ii) $Q_{m}=2 \mathrm{eV}$ and $t_{0}=4 \times 10^{-13} \mathrm{~s}$, and (iii) $Q_{m}=2.5 \mathrm{eV}$ and $t_{0}=4 \times 10^{-13} \mathrm{~s}$. The starting time $t_{0}=4 \times 10^{-13} \mathrm{~s}$ is in the range of the cascade duration $^{24}$ and consistent with the moleculardynamic simulation. ${ }^{25}$ The apparent activation in the first curve is $Q_{a}=0.08 \mathrm{eV}$, in the second curve $Q_{a}=0.1$ $\mathrm{eV}$, and in the third curve $Q_{a}=0.12 \mathrm{eV}$. The experimental data points were the growth temperature dependence of strains of Ge films grown with $200-\mathrm{eV} \mathrm{Ar}{ }^{+}$ion bombardment and ion-to-atom flux ratio, $J_{i} / J_{a}=0.02$. We can define a transition temperature, $T_{\text {tr }}$, above which the defect diffusion is thermally activated and below which the defect diffusion is cascade assisted. The transition

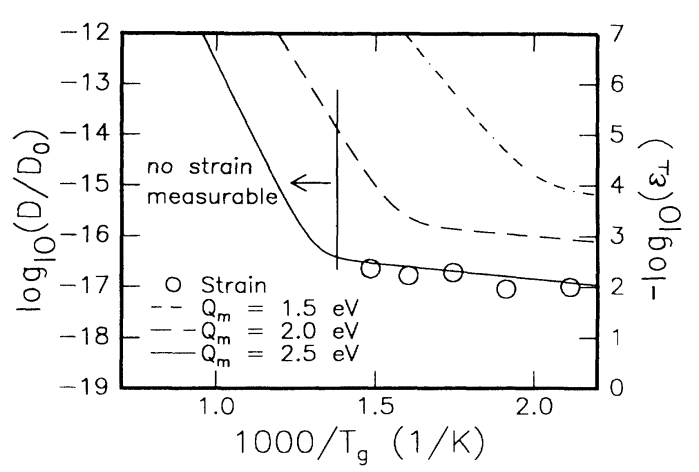

FIG. 4. Normalized diffusivity as a function of temperature for different activation energies of defect migration. All curves are calculated with $t_{0}=4 \times 10^{-13} \mathrm{~s}, F_{d}=150 \mathrm{eV} / \mathrm{nm}$, and $J_{i}=3 \times 10^{13}$ ions $/ \mathrm{cm}^{2}$-s. The experimental data points were the growth temperature dependence of strains of Ge films grown with $200-\mathrm{eV} \mathrm{Ar}^{+}$ion bombardment and ion-to-atom flux ratio, $J_{i} / J_{a}=0.02$.

temperature is written as

$$
T_{\mathrm{tr}}=\frac{Q_{m}}{k_{B}}\left[\ln \left(\frac{v}{\eta}\right)\right]^{-1} .
$$

As we can see from Fig. 4, the transition temperature for the third curve is about $800 \mathrm{~K}$. In previous work, we find that no strain can be detected for 200-eV $\mathrm{Ar}^{+}$. bombarded $\mathrm{Ge}$ films with an ion-to-atom flux ratio, $J_{i} / J_{a}=0.02$ at growth temperatures above $800 \mathrm{~K},{ }^{7}$ and that the defects are mobile at $800 \mathrm{~K}$ upon post-growth annealing of the strained films. ${ }^{8}$ Two points in Fig. 4 should be remarked: (i) the experimental data (strains) for value of $1000 / T_{g}$ below 1.4 was not measurable by the $\mathrm{x}$-ray rocking curve; and (ii) the experimental data points were intentionally shifted to lie on the third curve to show that the apparent activation energy obtained from the growth temperature dependence of the strains and the transition temperature are similar to the third curve. A fair agreement of both apparent activation energy and transition temperature between the experimental observation and the calculation was found to be $Q_{m}=2-2.5 \mathrm{eV}$ and $t_{0}=4 \times 10^{-13} \mathrm{~s}$. The corresponding pre-exponential factor is $2.32 \times 10^{-16} \mathrm{~cm}^{2} / \mathrm{s}$, which also agrees with the condition for the growth-rate limited regime. Note that the choice of $t_{0}$ and $Q_{m}$ is not unique. As $t_{0}$ decreases the apparent activation energy and $T_{\mathrm{tr}}$ decreases, and when $Q_{m}$ increases the apparent activation energy and $T_{\text {tr }}$ increase. Although the results of the calculation of the spike evolution is not exact due to the assumptions made and owing to the nonunique choice of $t_{0}$ and $Q_{m}$, the agreement between the experimental observation and this simple calculation suggests that the energy deposited by the energetic particle plays an important role in the defect diffusion process. Equation (4) has also been numerically integrated for finite $T_{g}$ by Gilmore, Haeri, and Sprague $^{26}$ to investigate the contribution of the thermal spike effect on the adatom surface diffusivity during ionbeam-assisted deposition of $\mathrm{Au}$ on $\mathrm{NaCl}$ using 100-eV 
$\mathrm{Ar}^{+}$ions. They concluded that the thermal spike would produce a negligible effect on adatom diffusivity for typical adatom densities. In contrast to the calculation of Gilmore, Haeri, and Sprague, we find that the thermal spike has a profound effect on the apparent activation energy of defect diffusion in spite of the small effective diffusivity.

Meyer et $a l .{ }^{27}$ have also obtained low activation energies, $0.15 \mathrm{eV}$ for $800-\mathrm{nm} \mathrm{Si}_{0.4} \mathrm{Ge}_{0.6}$ film and $0.5 \mathrm{eV}$ for 300-nm $\mathrm{Si}_{0.7} \mathrm{Ge}_{0.3}$ film grown on $\mathrm{Si}(100)$ by ion-beam sputtering deposition, from the growth temperature dependence of film stresses. Since the film thicknesses are above the critical thicknesses for dislocation formation, the films are expected to be at least partially relaxed. The low activation energy observed by Meyer et al. suggests that the major driving force for strain relaxation is the defect diffusion and the cascade-assisted diffusion is the dominant mechanism for defect migration.

We note also that Windischmann has provided a detail analysis of the ion peening model to explain the intrinsic stresses of thin films prepared by the ion-beam sputtering. ${ }^{28}$ The ion peening model, which is based on the knock-on linear cascade theory of transition sputtering proposed by Sigmund, ${ }^{29}$ can explain the intrinsic stresses for a variety of films deposited at a temperature where the defect mobility is sufficiently low that the growth conditions can be classified to be in the growth-rate limited regime and can provide a basis for the description of the total defect flux $K$. The combination of the ion peening model and the moving boundary diffusion model is straightforward and should be able to be used to analyze a variety of materials deposited with energetic ion beams involved.

\section{B. Ion incorporation}

The continuous moving boundary diffusion problem used in this analysis of defect-related strain produces parametric dependencies that are qualitatively similar to the discrete multisite model presented by $\mathrm{Ni}$ et al. ${ }^{14}$ Hence it seems reasonable to compare this analysis with the discrete model, which can be done by setting $K=\gamma J_{i}$ for dopant incorporation, where $\gamma$ is the incorporation coefficient which is the fraction of the ions which rest on or inside the target. The incorporation coefficient for fixed ion species and substrate is a function of ion energy and substrate temperature. In the case of $\mathrm{Sb}$ ion incorporation into $\mathrm{Si}$, the incorporation probability is assumed to be unity for all ion energies and for all substrate temperatures, i.e., $K=J_{i}$. We can also generalize the moving boundary diffusion model by additional terms in Eq. (1) which act to model dopant segregation. In the temperature regime used in the experiments of $\mathrm{Ni}$ et al. ${ }^{14}$ the diffusivity of the dopant at $900^{\circ} \mathrm{C}$ for $\mathrm{Sb}$ atoms in $\mathrm{Si}$ is comparable to $v R_{p}$, thus the dopant incorporation probability $\left(\mathrm{Cv} / J_{i}\right.$, the same as the definition of the bulk defects) will rise quickly at low growth rate and saturate. Figure 5 shows the data of incorporation probability of $\mathrm{Sb}$ atoms as a function of $\mathrm{Si}$ growth rate taken from Ref. 14 and the calculated curves using the moving boundary diffusion model. We find that the calculated curves can-

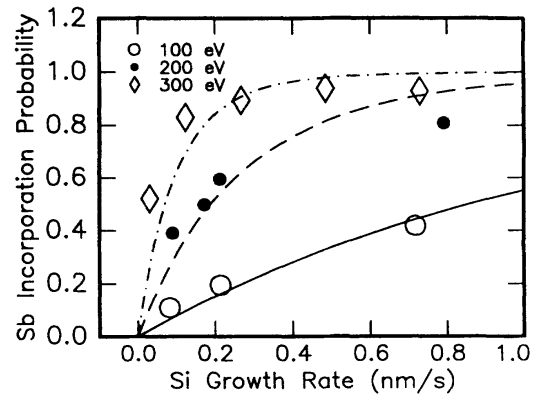

FIG. 5. Sb ions incorporation probability as a function of ion energy and the $\mathrm{Si}$ growth rate. The experimental data are taken from Ref. 14. The curves are calculated from Eq. (2ii) with fixed $R_{p} / \sqrt{2} \Delta R_{p}=2.357$ used in Ref. 14. The parameter for the solid line is $R_{p} / D=0.8 \mathrm{~s} / \mathrm{nm}$, for the dashed line $R_{p} / D=3.7$ $\mathrm{s} / \mathrm{nm}$, and for the dotted line $R_{p} / D=10 \mathrm{~s} / \mathrm{nm}$.

not fit the experimental data with a single activation energy. We follow the description in Ref. 14 to choose the $\mathrm{Sb}$ projected ranges for different energies, $R_{p}=0.25 \mathrm{~nm}$ for $100-\mathrm{eV}$ ions, $R_{p}=0.53 \mathrm{~nm}$ for $200-\mathrm{eV}$ ions, and $R_{p}=0.81 \mathrm{~nm}$ for $300-\mathrm{eV}$ ions, and fix $\Delta R_{p} / R_{p}=0.3$. The activation energies at $900^{\circ} \mathrm{C}$ obtained from $100-\mathrm{eV}$ Sb ion-beam data is $D=3.0 \times 10^{-15} \mathrm{~cm}^{2} / \mathrm{s}$, from $200-\mathrm{eV}$ $\mathrm{Sb}$ ion-beam data $D=1.4 \times 10^{-16} \mathrm{~cm}^{2} / \mathrm{s}$, and from 300 $\mathrm{eV} \mathrm{Sb}$ ion-beam data $D=8.1 \times 10^{-16} \mathrm{~cm}^{2} / \mathrm{s}$. Note that the diffusivities approach the bulk value as ion energies increase. The diffusivity values obtained lie in the range of those assumed for the third and fourth layers in Ni's model. The phenomenon of lower activation energy in the subsurface region suggests that the multisite model is necessary for dopant incorporation using low-energy ion beams.

The temperature dependence of the dopant incorporation has two cases: (i) the transition temperature between thermal diffusion and cascade-assisted diffusion is in the growth-rate-limited regime; and (ii) the transition temperature is in the diffusion-limited regime. From Eqs. (4) and (5), the number of jumps per unit time contributed from the spike for a defect is proportional to the ion flux $J_{i}$; thus, the transition temperature increases as the ion flux increases. For all other growth parameters fixed except the ion flux, case (i) corresponds to a low incoming ion flux and case (ii) corresponds to a high incoming ion flux. In case (i), the effective diffusivity dominated by the cascade-assisted diffusion at low temperature is so small such that $\alpha R_{p} \gg 1$. This indicated that the temperature dependence of the dopant incorporation would not be perturbed by the cascade-assisted diffusion, since the dopant incorporation process was already in the growthrate-limited regime. In case (ii), the cascade-assisted diffusion will dominate the diffusion process before the dopant incorporation process can be classified to be in the growth-rate-limited regime. This indicates that the effective diffusivity dominated by the cascade-assisted diffusion is so large such that $\alpha R_{p}$ is comparable to unity or much smaller than unity. Thus the approach of the dopant incorporation probability to unity is no longer controlled by the thermal activation energy; instead, is 


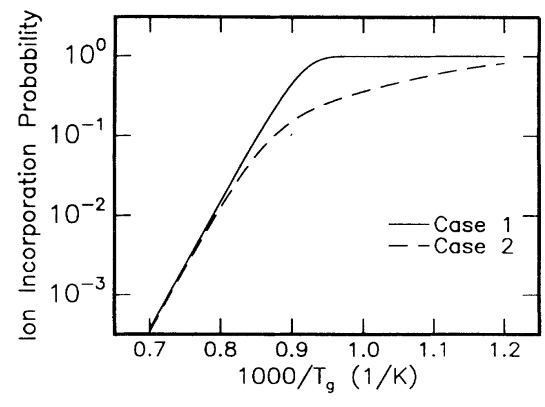

FIG. 6. The defect incorporation probability as a function growth temperature. In case 1 (solid line), the thermal diffusivity dominates from $\alpha R_{p}=0$ to $\alpha R_{p} \gg 1$. In case 2 (dashed line), the cascade-assisted diffusivity extends down to $\alpha R_{p}<1$. The parameters used to calculate these two curves are $D_{0}=0.214 \mathrm{~cm}^{2} / \mathrm{s}$ and $Q_{m}=3.2 \mathrm{eV}$ for thermal diffusivity, $D_{0}=1 \times 10^{-16} \mathrm{~cm}^{2} / \mathrm{s}$ and $Q_{a}=0.6 \mathrm{eV}$ for cascade-assisted diffusivity in case 1 , and $D_{0}=1 \times 10^{-13} \mathrm{~cm}^{2} / \mathrm{s}$ and $Q_{a}=0.6 \mathrm{eV}$ for cascade-assisted diffusivity in case 2 .

controlled by the apparent activation energy due to cascade-assisted diffusion below the transition temperature. The curve 1 in Fig. 6 shows case (i), in which the effect of cascade-assisted diffusion does not play an important role in dopant incorporation, and curve 2 corresponds to case (ii), in which a second activation energy which reflects the effective diffusivity due to cascadeassisted diffusion plays a role in the ion incorporation probability. The parameters used in calculating these two curves are shown in the figure caption. The only difference is a change in the effective pre-exponential factor for cascade-assisted diffusion which can be adjusted by changing the ion flux. The experimental conditions in Ref. 14 correspond to case (i) in Fig. 6 due to the low ion flux $\left(J_{S b}=4 \times 10^{7}-4 \times 10^{10}\right.$ ions $/ \mathrm{cm}^{2}$-s $)$ and high growth temperature $\left(T_{g}>600^{\circ} \mathrm{C}\right)$ which were used. Figure 7 shows the data points taken from Ref. 14 and the curves calculated using Eq. (2ii) with $v=0.17 \mathrm{~nm} / \mathrm{s}, R_{p}=1.1$

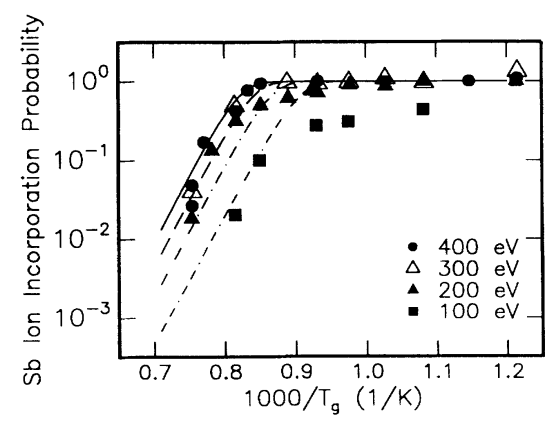

FIG. 7. The temperature dependence of the dopant incorporation for different $\mathrm{Sb}$ ion energies. The data points are taken from Ref. 14. All lines are calculated with $D_{0}=0.214 \mathrm{~cm}^{2} / \mathrm{s}$ and $v=0.17 \mathrm{~nm} / \mathrm{s}$. The values of $R_{p}, 0.25,0.53,0.81$, and 1.1 $\mathrm{nm}$, and the values of activation energy for Sb ions in $\mathrm{Si}, 3.23 \mathrm{eV}$ (dot-dashed-dashed line), $3.31 \mathrm{eV}$ (dot-dashed line), $3.36 \mathrm{eV}$ (dashed line), and $3.40 \mathrm{eV}$ (solid line), correspond to 100, 200, 300 , and $400 \mathrm{eV} \mathrm{Sb}$ ions, respectively. $\mathrm{nm}$ for $400-\mathrm{eV} \mathrm{Sb}$ ions, and the choices of $R_{p}$ at other different energies are the same as before. $D_{0}=0.214$ $\mathrm{cm}^{2} / \mathrm{s}$ for all energies, the activation energy is $3.23 \mathrm{eV}$ for $100-\mathrm{eV} \mathrm{Sb}$ ions, $3.31 \mathrm{eV}$ for $200-\mathrm{eV} \mathrm{Sb}$ ions, $3.36 \mathrm{eV}$ for $300-\mathrm{eV} \mathrm{Sb}$ ions, and $3.40 \mathrm{eV}$ for $400-\mathrm{eV} \mathrm{Sb}$ ions. The activation energies for thermal diffusion are chosen this way so that the previous diffusivities at $900^{\circ} \mathrm{C}$ for different ion energies, obtained from the growth-ratedependence of dopant incorporation are numerically equal in the context of the present single activation energy model. The calculated curves fit the data points well for ion energies larger than $300 \mathrm{eV}$ while giving poor fits for lower energy ion beams. It is also interesting to compare the difference between the dopant and inert gas incorporation. Our previous measurements of the Xe concentration trapped inside $\mathrm{Ge}$ films ${ }^{7}$ indicated that the incorporation probability of $\mathrm{Xe}$ was only about $3 \%$ even when the growth conditions were in the growth-ratelimited regime. This suggests the importance of the incorporation coefficient.

The major deviation of the moving boundary diffusion model from low-energy ion incorporation data is due to the multiple activation processes for dopant incorporation such as adsorption and desorption of the ions at the surface, the difference between surface phonon and bulk phonon, the electronic state near surface, and the thermal spike effect which all could contribute to the multiple activation processes. All the curves in Figs. 6 and 7 are calculated from Eq. (2ii), which is a solution subjected to the boundary condition that $N(0)=0$, which is not a proper condition for low-energy dopant incorporation processes and predicts that no dopant incorporation will occur through adsorption and desorption processes. Since the simple moving boundary diffusion model neglects the depth dependence of the diffusivity of the defects, the quantitative use of this simple model is only suitable for higher energy ion beams (e.g., $E_{i}>300 \mathrm{eV}$ for Sb ions incorporation into $\mathrm{Si}$ ).

\section{CONCLUSIONS}

The IAMBE processes can be modeled using a continuous moving boundary diffusion model. The defect concentrations generated by the ion beam are proportional to the ion-to-atom flux ratio in the growth-rate-limited regime. The definitions of surface defects (defects that will come out to the surface) and bulk defects (defects that will be trapped inside the bulk) were defined in a dynamic term as a function of the growth rate, defect production range, and defect diffusivity. The low apparent activation energy obtained from the growth temperature dependence of the strain annihilation is explained by cascadeassisted defect diffusion. In IAMBE processes, the ion beam generates a cascade which undergoes athermal and thermal recovery. The defects left after cascade recovery have a diffusivity determined by the relative importance of the thermal diffusion and subsequent cascade-assisted diffusion. The same equation can also be used to describe the dopant incorporation processes and reasonable agreement with experimental data can be found for higher ion energies. In low-energy ion incorporation processes, the 
defect incorporation may accompany the dopant incorporation. In general, the activation energies for defect diffusion and dopant diffusion are not the same. Neglecting the interaction between defects and dopants, one could define transition temperatures for both defects and dopants. For enhancing dopant incorporation while minimizing defect incorporation, one should also avoid the dopant incorporation processes at the temperature regime below $T_{\mathrm{tr}}$, where the defects generated by the ion beam are stable. The moving boundary diffusion model gives a unified view of dopant and defect incorporation processes. The multiple activation site in the surface region can be done by adding layers in the surface region with different diffusivities and continuous boundary con- ditions at each interface. However, the model can only be used to describe the incorporation of pointlike defects. When extended defects formation or the segregation of the dopants are involved, the simple model needs to be modified.

\section{ACKNOWLEDGMENTS}

We would like to thank Dr. A. Mutz for valuable discussions. One of us (C.J.T.) also acknowledges the support of the IBM corporation. This work was supported by National Science Foundation Grant Nos. DMR8958070 and DMR-8811795, and the A.T. \& T. Foundation.
* Present address: Gordon McKay Laboratory of Applied Science, Harvard University, Cambridge, MA 02138.

1J. E. Greene, CRC Crit. Rev. Solid State Mater. Sci. 2, 47 (1983).

2J. Y. Tsao, E. Chason, K. M. Horn, D. K. Brice, and S. T. Picraux, Nucl. Instrum. Methods B 39, 72 (1989).

${ }^{3}$ E. Chason, J. Y. Tsao, K. M. Horn, and S. T. Picraux, J. Vac. Sci. Technol. B 7, 332 (1989).

${ }^{4}$ E. Chason, J. Y. Tsao, K. M. Horn, S. T. Picraux, and H. A. Atwater, J. Vac. Sci. Technol. A 8, 2507 (1990).

${ }^{5}$ K. M. Horn, J. Y. Tsao, E. Chason, D. K. Brice, and S. T. Picraux, J. Appl. Phys. 69, 243 (1991).

${ }^{6}$ C.-H. Choi, L. Hultman, and S. A. Barnett, J. Vac. Sci. Technol. A 3, 1587 (1990).

${ }^{7}$ C. J. Tsai, H. A. Atwater, and T. Vreeland, Appl. Phys. Lett. 57, 2305 (1990).

${ }^{8}$ C. J. Tsai, P. Rozenak, H. A. Atwater, and T. Vreeland, J. Cryst. Growth 111, 931 (1991).

${ }^{9}$ P. C. Zalm and L. J. Beckers, Appl. Phys. Lett. 41, 167 (1982).

${ }^{10}$ T. Itoh, T. Nakamuru, M. Muromachi, and T. Sugiyama, Jpn. J. Appl. Phys. 16, 553 (1977).

${ }^{11}$ C. Schwebel, F. Meyer, G. Gautherin, and C. Pellet, J. Vac. Sci. Technol. B 4, 1153 (1986).

${ }^{12}$ F. Meyer, C. Schwebel, C. Pellet, G. Gautherin, A. Buxbaum, M. Eizenberg, and A. Raizman, Thin Solid Films 184, 117 (1990).

${ }^{13}$ T. Ohmi, K. Hashimoto, M. Morita, and T. Shibata, J. Appl. Phys. 69, 2062 (1991).
${ }^{14}$ W.-X. Ni, J. Knall, M. A. Hasan, G. V. Hansson, J.-E. Sundgren, S. A. Barnett, L. C. Markert, and J. E. Greene, Phys. Rev. B 40, 10449 (1989).

${ }^{15}$ W. J. Bartels, J. Vac. Sci. Technol. B 1, 338 (1983).

${ }^{16}$ C. R. Wie, T. A. Tombrello, and T. Vreeland, J. Appl. Phys. 59, 3743 (1986).

${ }^{17}$ A. K. Myers-Beaghton and D. D. Vvedensky, Phys. Rev. B 42, 5544 (1990).

${ }^{18}$ R. Kelly, Radiat. Eff. 32, 91 (1977).

${ }^{19}$ P. Sigmund and C. Claussen, J. Appl. Phys. 52, 990 (1981).

${ }^{20}$ G. J. Dienes and G. H. Vineyard, Radiation Effects in Solids (Interscience, New York, 1957); G. H. Vineyard, Radiat. Eff. 29, 245 (1976).

${ }^{21}$ Y. T. Cheng, Mater. Sci. Rep. 5, 47 (1990).

${ }^{22}$ I. Barin, Thermochemical Data of Pure Substances (VCH, New York, 1989), Pt. 1.

${ }^{23}$ M. W. Thompson and R. S. Nelson, Philos. Mag. 7, 2015 (1962).

${ }^{24}$ M. W. Thompson, Defects and Radiation Damage in Metals (Cambridge University Press, London, 1969).

${ }^{25}$ R. S. Averback, Nucl. Instrum. Methods B 15, 675 (1986).

${ }^{26}$ C. M. Gilmore, A. Haeri, and J. A. Sprague, Thin Solid Films 165, 359, 1988.

${ }^{27}$ F. Meyer, M. Zafrany, M. Eizenberg, R. Beserman, C. Schwebel, and C. Pellet, J. Appl. Phys. 70, 4268 (1991).

${ }^{28}$ H. Windischmann, J. Appl. Phys. 62, 1800 (1987).

${ }^{29} \mathrm{P}$. Sigmund, in Sputtering by Particle Bombardment I, edited by R. Behrisch (Springer, Berlin, 1981), Chap. 2. 